\title{
Planung, Vorbereitung und Kontrolle bei Eingriffen im Bereich der HWS
}

\author{
Thomas Weiß, Oliver Gonschorek, Stefan Hauck, Volker Bühren
}

\section{Zusammenfassung}

Die Planung und Vorbereitung von operativen Eingriffen an der HWS muss präzise und exakt indiziert werden. Die Schwierigkeit besteht dabei in der Vielzahl an Verletzungsmustern, die insbesondere an der oberen HWS auftreten. Der 1. Schritt im Rahmen der Vorbereitung ist zunächst eine adäquate Bildgebung, meist durch schnittbildgebende Verfahren. Bei Unklarheiten werden diese durch eine Funktionsdiagnostik ergänzt. Die korrekte Klassifikation und somit die Zuordnung zu einer instabilen, hoch instabilen bzw. stabilen Verletzung sollte damit ermöglicht werden. Die Entscheidung zur weiteren Versorgung, ob operativ oder konservativ, resultiert aus weiteren Kofaktoren, die das Alter, die Knochenqualität, den Anspruch des Patienten sowie das Komplikationsrisiko, insbesondere von Schraubenfehllagen berücksichtigen müssen. Wird der Entschluss zur operativen Versorgung gefasst, muss eine sinnvolle Lagerung und Repositionsmöglichkeit im Vorfeld geplant werden. Hierzu zählen verschiedene Tools, die im Folgenden dargestellt werden. Die besondere Anatomie der HWS mit der Nähe zum Rückenmark, den Gefäßen sowie den Halsorganen und ihrer grazilen Struktur erfordert exakte anatomische Kenntisse sowie eine präzise handwerkliche Ausführung. Ein großer Fortschritt sind hier neue bildgebende Verfahren, die eine bisher nicht bekannte intraoperative Sicherheit und Kontrolle bieten. Hierzu zählt die intraoperative Schnittbilddiagnostik mit sagittalen und koronaren Rekonstruktionen, weiterhin die Möglichkeit zur Navigation mit optoelektronisch gesteuerten Instrumenten auf der Grundlage der CT-Darstellung mit Trackern. Die intraoperative Kontrolle wird weiterhin erhöht durch mikrochirurgische und minimalinvasive Verfahren sowie die Verwendung eines Operationsmikroskops.

\section{Planning, Preparation and Control of Interventions in the Region of the Cervical Spine}

The planning of cervical spine surgery must be exactly indicated and precise in its performance. The difficulties are the variety of different injuries of the cervical spine, especially the upper cervical spine. The first step in planning cervical spine surgery must be an adequate radiological imaging, in most cases with spiral CT imaging and MRI. In cases with segmental instability a functional diagnostic work-up is important. With this information it should be possible to find the right classification for the cervical spine in- jury and to decide between stable, unstable and highly unstable lesions. The indication for surgery not only depends on the presence of stable or unstable lesions, it is also the result of co-factors like age, bone quality, patient's life style and the risk of complications such as wrong screw placement. If the decision for surgery is clear, the surgeon should think about the possibilities for repositioning and a good set-up in the OR. The different tools required are explained in this article. The special anatomy of the cervical spine with the direct neighborhood of vessels, the cervical organs, the dura and the gracile structure of the bones require a very good knowledge of anatomy and a precise technique of surgery. Major progresses in intraoperative control and safety are provided by the new imaging devices. Intraoperative spiral $\mathrm{CT}$ imaging with coronary and sagittal reconstructions give a high quality of imaging and safety for screw placement. Additionally, the possibility for navigation with optoelectronically controlled instruments is very safe. As reference it is necessary to place a tracker near the operation field and perform a CT scan. Other new means for controlled cervical spine surgery are the microscope, minimally invasive surgery and microsurgery.

\section{Einleitung}

Die Planung bei Eingriffen im Bereich der Halswirbelsäule stellt eine Besonderheit dar angesichts der Vielzahl an Verletzungsbildern und Erkrankungen der Halswirbelsäule. Es soll im Folgenden

OP-JOURNAL 2011; 27: 8-17

(c) Georg Thieme Verlag KG Stuttgart · New York DOI http://dx.doi.org/10.1055/s-0030-1271029 die diagnostische Vorbereitung zur korrekten Therapieeinleitung dargestellt werden.

Hierzu ist bereits die Klassifikation für die einzelnen HWS-Regionen eine Besonderheit, weil die Klassifikation an der oberen HWS von der allgemein gültigen Klassifikation nach Magerl [1-3,11] abweicht. Sie soll im Folgenden nochmals dargestellt werden.
Diagnostische Besonderheiten wie die Funktionsdiagnostik sind an der HWS meist erforderlich aufgrund häufiger diskoligamentärer Verletzungen. Diese werden im Einzelnen dargelegt. Je nach Verletzungsschwere bzw. Dislokationsgrad sind spezielle Repositionsmanöver und Lagerungstechniken erforderlich. Ein Beispiel sind die verhakten Luxationen.

Weitaus häufiger werden Eingriffe an der HWS jedoch aufgrund degenerativer 
Erkrankungen durchgeführt. Hierzu ist insbesondere eine exakte klinische Untersuchung zwingend erforderlich. Bei neurologisch unauffälligen Patienten sollte immer ein konservativer Therapieversuch vorangestellt werden.

Die Entscheidungsfindung beim traumatologischen Patientengut mit instabilen Verletzungen ist demgegenüber vergleichsweise einfach.

Die Kontrolle bei HWS-Eingriffen muss möglichst präzise sein. Zur spinalen Dekompression werden mikrochirurgische Techniken angewandt. Die Schraubenplatzierung erfordert besondere Kenntnisse der Anatomie wie auch eine präzise handwerkliche Ausführung. Präoperativ sollte die Schraubenplatzierung am CT geplant werden. Hierzu zählt die Ausmessung der Schraubenlänge und Stärke wie auch die Konvergenz bzw. Divergenz bei Massa-lateralis-Schrauben. Insbesondere Pedikelschrauben benötigen eine deutlich höhere Konvergenz im Vergleich zur oberen BWS. Bei C2/1-Instrumentierungen ist eine vorherige Lagebestimmung des Foramen transversarium absolut indiziert, um hohe Abbiegungen im Corpus C2 zu erkennen. In bis zu 20\% der Fälle sind Gelenkschrauben C2/1 dann nicht zu platzieren [12]. Eine Röntgenkontrolle im OP ist obligat, die Schwierigkeit besteht jedoch in der mangelnden Darstellbarkeit der oberen wie auch der unteren HWS. Eine Verbesserung der Kontrolle ergibt sich durch die Möglichkeit der Navigation sowie der intraoperativen CT-Darstellung. Dies soll im Folgenden anhand des O-Arms ${ }^{\circledR}$ (Medtronic GmbH, Meerbusch) dargestellt werden.

\section{Klassifikation}

Die Besonderheit der HWS besteht in ihrer Aufteilung in obere $(\mathrm{CO}-\mathrm{C} 2)$ und untere HWS (C3-C7). Die obere HWS ist hierbei insbesondere für die Rotation im C1/2-Gelenk verantwortlich. Demgegenüber ist die untere HWS für Flexion und Extension verantwortlich [13].

Die Klassifikation der unteren HWS richtet sich nach der üblichen Magerl-Klassifikation der restlichen Wirbelsäule. Instabile Verletzungen sind hierbei Bund C-Verletzungen als Flexions-/Distraktionsverletzungen bzw. rotatorische Instabilitäten. Diese sind per se instabil und somit der operativen Therapie zuzuführen. Verletzungen der vorderen Säule (A-Verletzungen) sind je nach Stabilität der Hinterkante bzw. Kyphosewinkel konservativ versus operativ zu therapieren.

Eine Besonderheit ist jedoch die Bandscheibenzerreißung, da sie an der HWS häufiger auftritt und oft übersehen werden kann (MRT!).

Die Klassifikation der oberen HWS zeichnet sich durch eine Vielzahl an Verletzungsbildern aus. Diese sind jeweils in einer Klassifikation dargestellt:

\section{Frakturen der Okzipitalkondylen}

Die Einteilung erfolgt nach Jeanneret [13].

Typ 1: Schädelbasisfraktur vom Foramen magnum ausgehend und durch eine Okzipitalkondyl laufend

Typ 2: Schädelbasisringfraktur, bisher nur bei Verkehrstoten im Rahmen der Obduktion nachgewiesen

Typ 3: einseitige Kompressionsfraktur einer Okzipitalkondyle

Typ 4: Abrissfraktur der Ligg. Alaria, in etwa ein Viertel der Fälle in Kombination mit einer kompletten oder inkompletten atlantookzipitalen Dislokation (AOD)

Die Therapie der Wahl ist praktisch immer konservativ. Lediglich bei Vorliegen einer AOD (falls diese überlebt wird) wird eine operative Spondylodese von dorsal CO-C4 durchgeführt.

\section{Atlantookzipitale Dislokation (AOD)}

Die Verletzung ist praktisch immer tödlich und wird nur durch eine kurze Rettungskette ggf. radiologisch diagnostiziert. Eine Therapieeinleitung ist daher meist nicht notwendig. Häufig sind Kinder von dieser Verletzung betroffen.

Die Einteilung erfolgt nach Traynelis [13] und beschreibt die Dislokationsrichtung.

Typ 1: ventrale Dislokation (häufig)

Typ 2: dorsale Luxation

Typ 3: axiale Dislokation, meist in Kombination mit ventraler Dislokation

Für dieses hoch instabile Patientengut kommt in der Anfangsphase nur die Ruhigstellung im Halofixateur in Betracht. Eine weitere Distraktion muss hierbei vermieden werden. Nach Stabilisierung des Patienten ist ggf. eine dorsale Fusion einzuleiten.

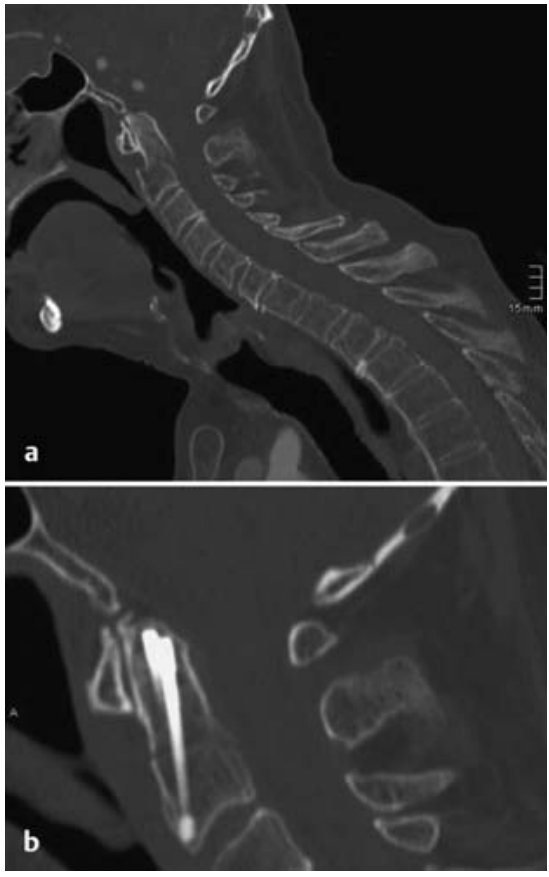

Abb. $1 \mathbf{a}$ und $\mathbf{b}$ Typische dislozierte Andersson-II-Extensionsfraktur. Die ventrale Verschraubung wird über 2 Doppelgewindeschrauben in kanülierter Technik $(2,8 \mathrm{~mm})$ durchgeführt. Die Reposition musste über ein HWS-Aggregat gehalten werden.

\section{Atlantoaxiale Dislokation (AAD)}

Eine Unterteilung erfolgt in translatorische und rotatorische Instabilitäten. Die translatorische Verschiebung wird analog zur AOD meist nicht überlebt. Die rotatorische Verschiebung betrifft meist Jugendliche und zeichnet sich durch eine typische Kopfhaltung aus. Eine Reposition muss daher in Narkose eingeleitet werden. Eine weitere Ruhigstellung im Halo bzw. Minervagips ist meist ausreichend.

\section{Densfrakturen}

Die Densfraktur ist eine typische Wirbelsäulenverletzung des älteren Menschen.

Darüber hinaus machen Densfrakturen über die Hälfte von HWK-2-Frakturen aus.

Die Einteilung erfolgt rein morphologisch-anatomisch in 3 Typen nach Anderson und D'Alonzo [13].

Ergänzend zu dieser Einteilung findet sich ein Unterschied in der operativen Therapie der Andersson-2-Frakturen bei Flexions- und Extensionsverletzungen. Die Versorgung bei Flexionsverletzungen kann aufgrund des Frakturverlaufs nicht 
von ventral erfolgen, sondern sollte von dorsal durch Gelenkschrauben stabilisiert werden (s. auch Fallbsp.) Extensionsverletzungen können demgegenüber von ventral stabilisiert werden (Abb. 1).

Typ 1: Fraktur der Densspitze. Entspricht im Wesentlichen einem Ausriss der Ligg. Alaria.

Typ 2: Fraktur des Processus odontoideus oberhalb seiner Basis. Sie stellt die häufigste Frakturform dar und ist vergesellschaftet mit einer hohen Pseudarthrosenrate aufgrund der hohen Dislokationstendenz.

Typ 3: Frakturverlauf im Axiskörper. Es handelt sich um breitbasige knöcherne Verletzungen der Densbasis und ein konservatives Therapieregime ist somit ausreichend

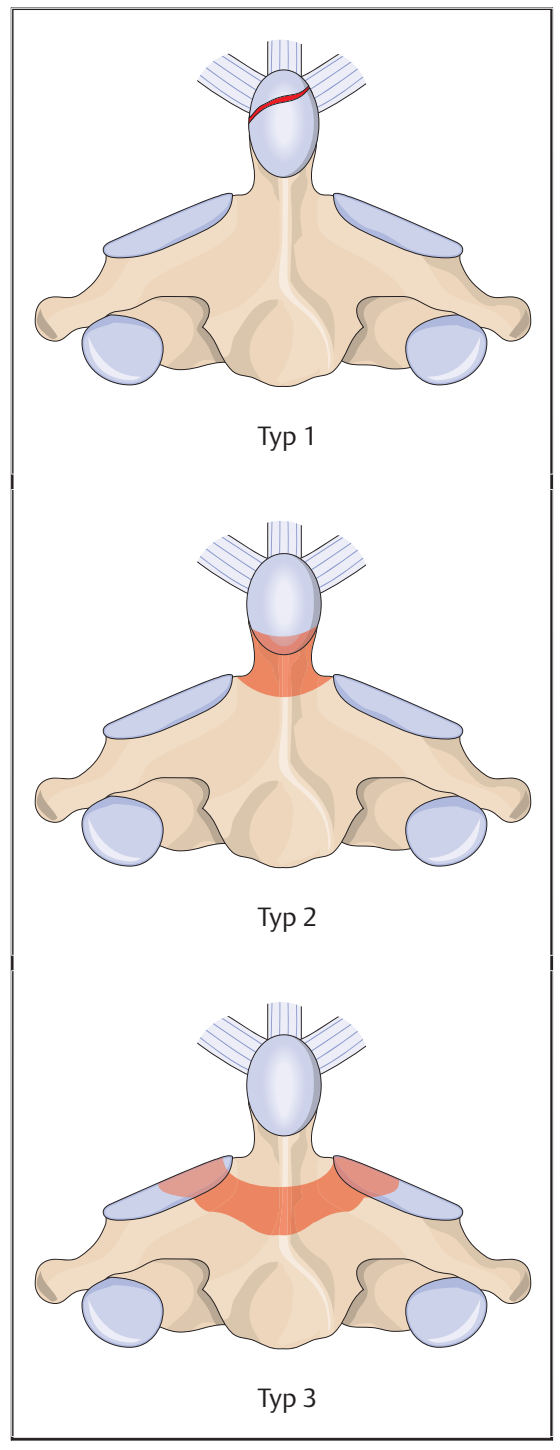

\section{Atlasfrakturen}

Atlasfrakturen entstehen durch eine Kombination aus Kompression und Hyperextension. Es werden 5 Typen unterschieden, wobei nur einer der operativen Therapie zugeführt werden muss.

Typ 1: isolierte Fraktur des vorderen Atlasbogens (stabil)

Typ 2: isolierte Fraktur des hinteren Atlasbogens, immer doppelseitig (stabil)

Cave: differenzialdiagnostisch oft auch Bogenschlussanomalien

Typ 3: Jefferson-Fraktur: komplette Berstung des vorderen und hinteren Atlasbogens (bei Ruptur des Lig. transversum instabil!)

Typ 4: isolierte Fraktur einer Massa lateralis (stabil)

Typ 5: isolierte Fraktur des Proc. transversus (stabil)

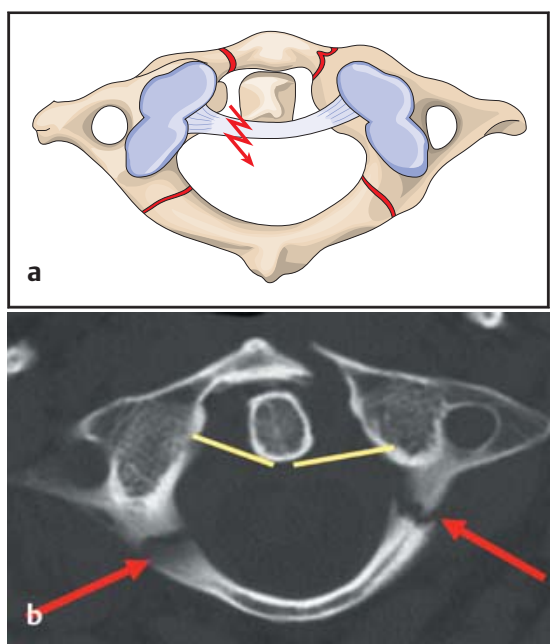

Jeffersonfraktur.

\section{Traumatische Spondylolisthesis des Axis}

Die bekannteste Spondylolisthese HWK 2/3 ist die Hangman-Fraktur. Sie stellt auch den einzig wirklich instabilen Typ dar, der einer operativen Spondylodese bedarf. Die Einteilung erfolgt nach Effendi 1-3 [5]. Beim Typ 1 ist die Bandscheibe nicht verletzt und die Bögen sind geringst disloziert. Beim Typ 2 ist demgegenüber die Bandscheibe verletzt, die Gelenke dorsal sind jedoch unbeteiligt. Beim Typ 3 (Hangman) sind zusätzlich die Gelenke disloziert.

Diese Verletzung muss operativ mittels ventraler Spondylodese stabilisiert werden [5].

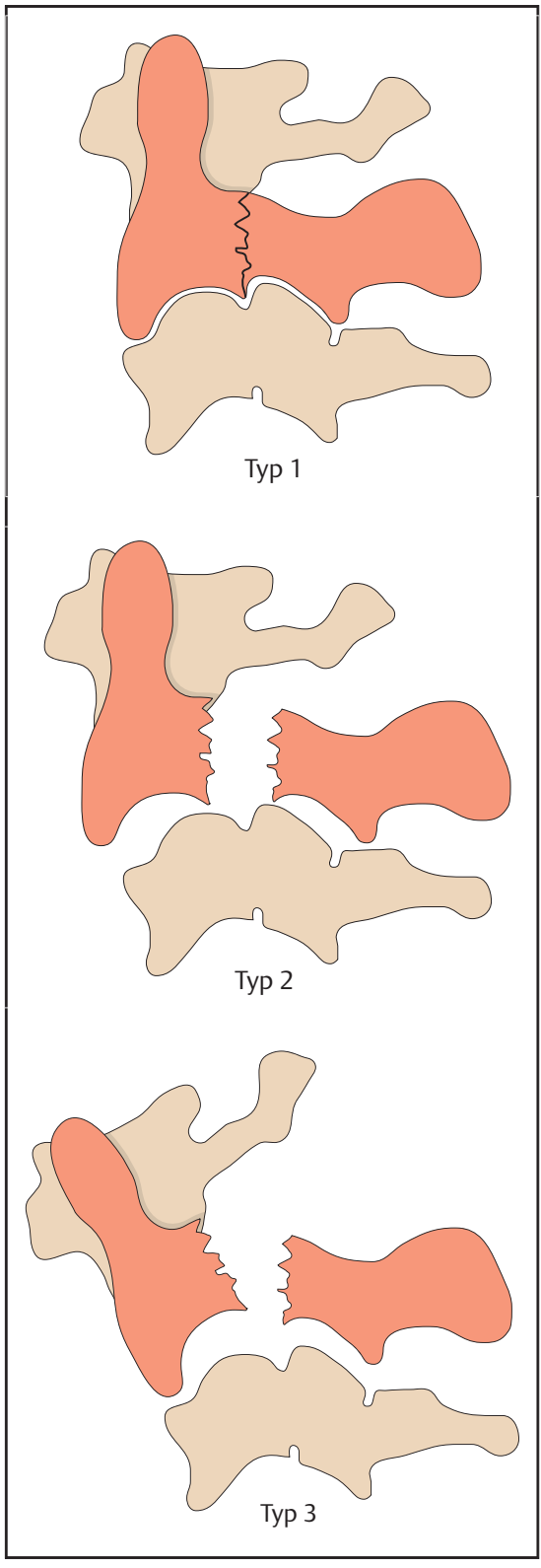

\section{Diagnostik}

Die 3 Pfeiler der Diagnosik sind Anamnese, körperliche Untersuchung und gezielte radiologischen Diagnosik.

Es soll im Folgenden auf die Besonderheiten der radiologischen Bildgebung eingegangen werden.

\section{Röntgen}

Die Grundlage ist sicherlich die konventionelle radiologische Diagnostik. Hierzu zählt an der HWS die seitliche Aufnahme, die a.-p. Aufnahme der unteren HWS sowie die transorale a.-p. Aufnahme von Atlas und Axis. Die HWS muss dabei im seitlichen Strahlengang bis einschließlich C7/Th1 dargestellt werden. 


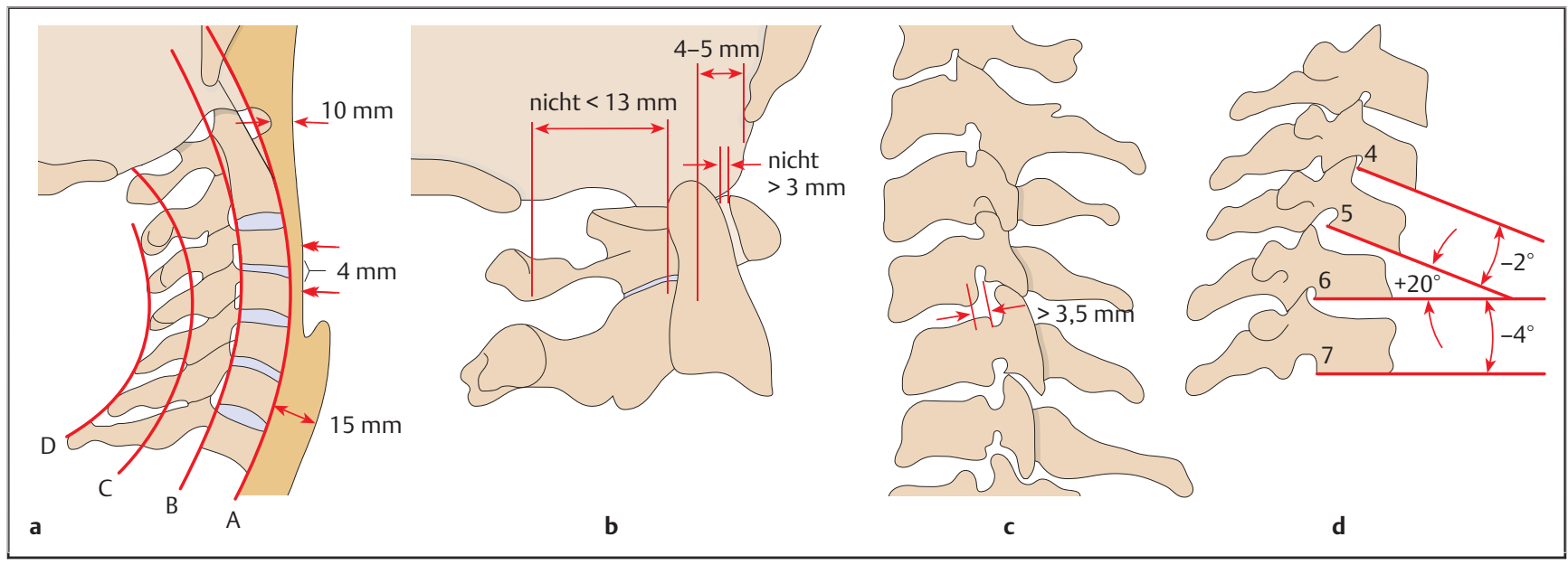

Hilfslinien im Röntgenbild. a Hilfslinien im seitlichen Röntgenbild zur Bestimmung des Alignements. b Grenzdistanzen an der oberen HWS im seitlichen Röntgenbild. c Maximal tolerable Translation im seitlichen Röntgenbild. d Maximal tolerable Kyphose im seitlichen Röntgenbild*.

* Abb. modifiziert nach: Ulrich C, Bühren V. Verletzungen der Halswirbelsäule. Orhtopädie und Unfallchirurgie up2date 2006; 1: 415-446 [13]

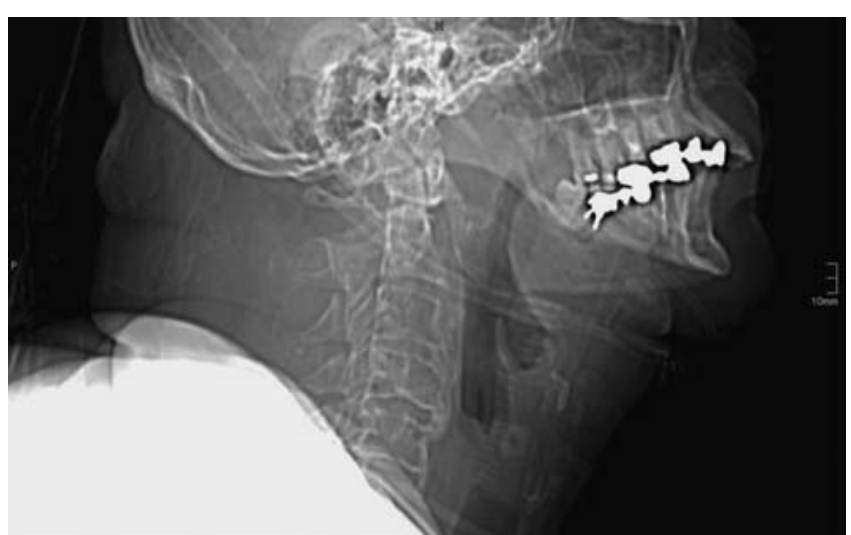

Abb. 2 Die seitliche Nativaufnahme ist lediglich bis HWK 5 dargestellt.

Nur so lassen sich $90-95 \%$ aller Verletzungen darstellen. Der Verletzungsschwerpunkt an der HWS liegt im zervikothorakalen Übergangsbereich. Ist dieser Bereich im konventionellen Bild nicht komplett dargestellt, so muss ein CT zur Komplettierung nachgefahren werden (Abb. 2, 3). Meist ist die HWS unvollständig im unteren Abschnitt abgebildet durch Überlagerung des Schultergürtels. Es ist deshalb wichtig, dass die Schultern beim liegenden Patienten an den Armen heruntergezogen werden. Die früher durchgeführte Schwimmer-/ Fechteraufnahme ist als veraltet zu betrachten und im Zeitalter der CT-Diagnostik obsolet.

Zur Beurteilung der Standarddiagnostik sind Hilfslinien und standardisierte Messwerte zur Beurteilung heranzuziehen [13].
CT

Goldstandard einer HWS-Verletzung ist die Spiral-CT-Untersuchung mit multiplanarer Rekonstruktion. Insbesondere im zervikothorakalen Übergang werden Verletzungen eindeutig dargestellt. Eine hohe Sicherheit in der Diagnosik erhält man durch sagittale und koronare Rekonstruktionen. Oft werden Fehlstellungen erst dort diagnostiziert (Abb. 4-6).

In Abhängigkeit der Fragestellung bei zervikalen Bandscheibenproblemen sind Dünnschicht-CTs durchzuführen, um die spinale bzw. neuroforaminale Enge einzuschätzen. Insbesondere bei lateralen Bandscheibensequestern und knöchernen Stenosen sind Dünnschichtsequenzen indiziert (zusätzlich zum MRT).

Die Durchführung von Rekonstruktionen birgt jedoch auch Risiken. So können insbesondere im kraniozervikalen Übergang Densfrakturen vorgetäuscht werden, einerseits durch Überlagerung und

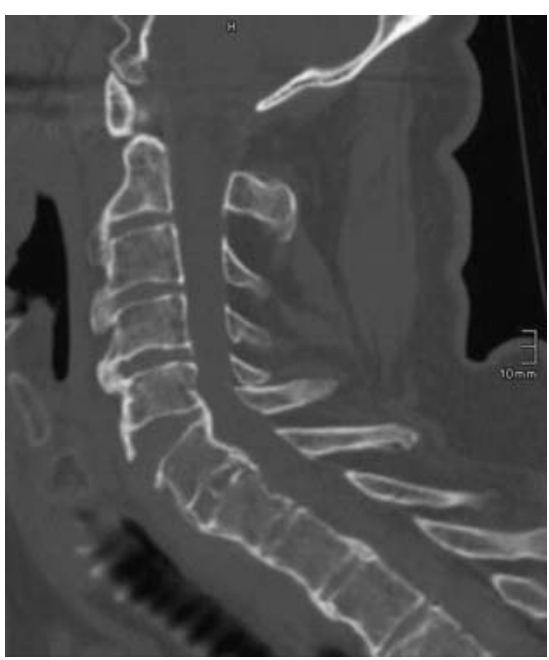

Abb. 3 Erst die CT-Diagnostik ergibt die ventral klaffende Fraktur bei Ankylose der Wirbelsäule.

Artefaktbildung bei Zahnimplantaten und Provisorien wie auch durch Verwackeln in den entscheidenden Schichten der HWK-2-Region. Vermutlich liegt eine Artefaktbildung auch durch den Schluckakt (Abb. 7-9) vor. Diagnostische Sicherheit ergibt sich nur durch Wiederholung der CT-Sequenz. Erkennbar ist die Artefaktbildung anhand des Pixelversatzes, der sich nach dorsal projiziert.

\section{MRT}

Die Vorzüge der Kernspintomografie liegen in der Darstellung der Weichteilstrukturen und der strahlungsfreien Bildgebung. Weitere Vorteile sind die Darstellbarkeit auch längerer Wirbelsäulenabschnitte bis zur Abbildung der gesamten Wirbelsäule, um Mehretagenver- 


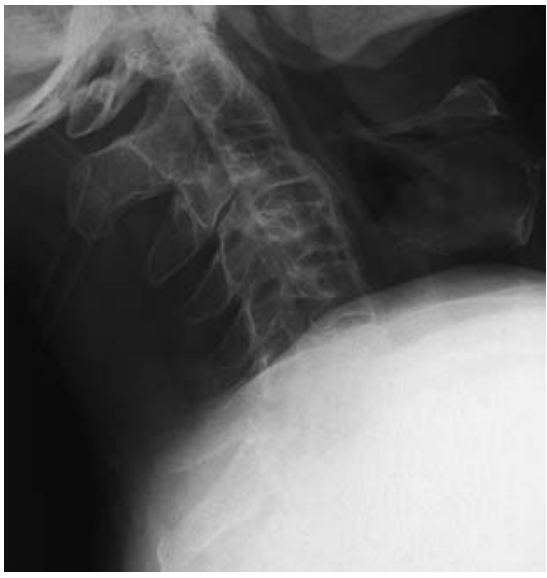

Abb.4 Der zervikothorakale Übergang ist nicht mit abgebildet.

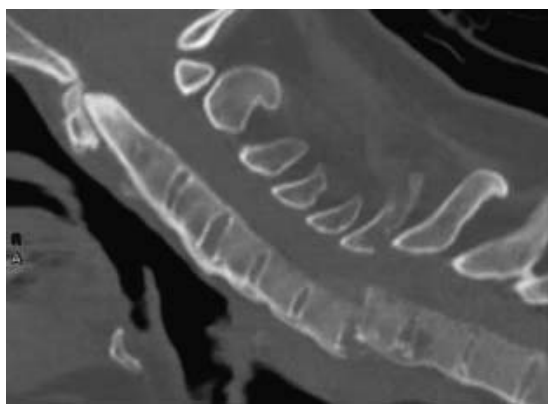

Abb. 5 Die CT-Diagnostik zeigt die komplette Luxation C6/7 beim Bechterew-Patienten.

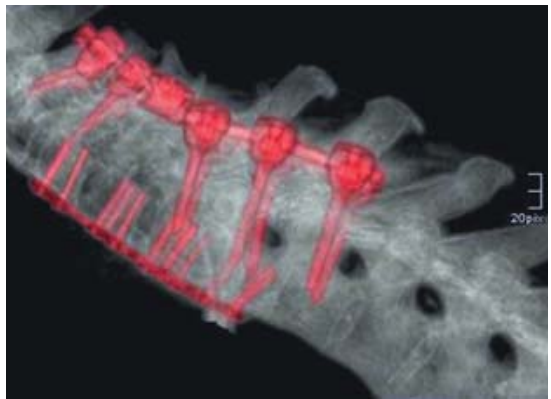

Abb. 6 Die operative Stabilisierung muss dahingehend sehr stabil gewählt werden mit dorsoventraler langstreckiger Instrumentierung.

letzungen darzustellen. Die bevorzugte Indikation ist der Nachweis einer Bandscheibenschädigung und die ligamentäre Zerreißung (Abb.10-12). Bei Vorliegen von neurologischen Symptomen und negativem CT-Befund ist eine MRT zu fordern, um Schädigungen der Weichteilstrukturen nachzuweisen. Bleibt auch die MRT-Diagnostik unauffällig, so liegt ein sog. SCIWORA-Syndrom vor (spinal cord injury without radiologic abnormality) (Abb. 13).

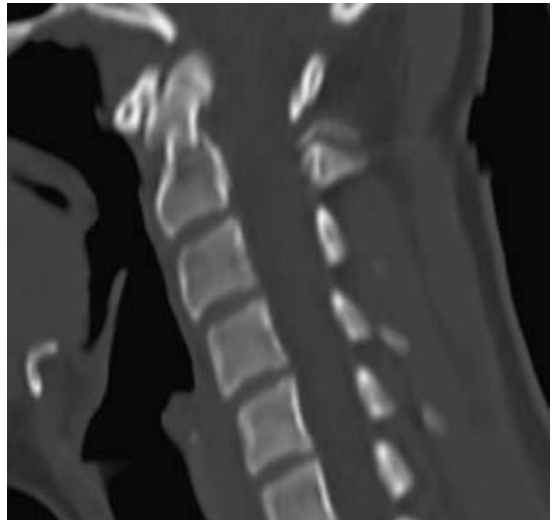

Abb. 7 Eine jugendliche Patientin nach Reitunfall mit initialer Neurologie wird mit der Diagnose Densfraktur zuverlegt zur operativen Versorgung.

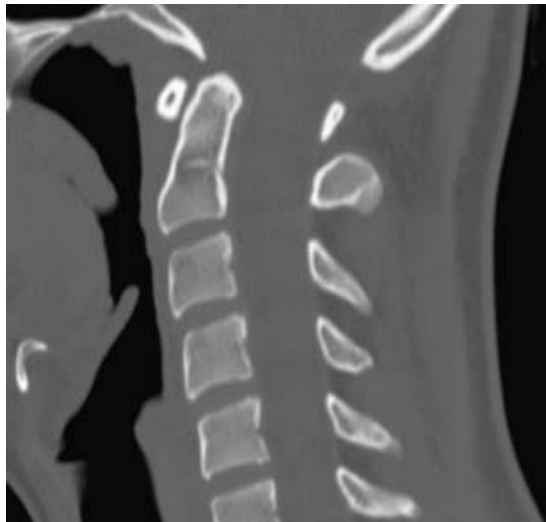

Abb. 8 Nach Erkennnung des Artefakts wird eine Kontroll-CT-Darstellung durchgeführt mit der Diagnose eines Normalbefunds. Vermutlich hat die Patientin im entscheidenden Moment gewackelt. Denkbar wäre auch eine $\mathrm{Ar}$ tefaktbildung durch den Schluckakt.
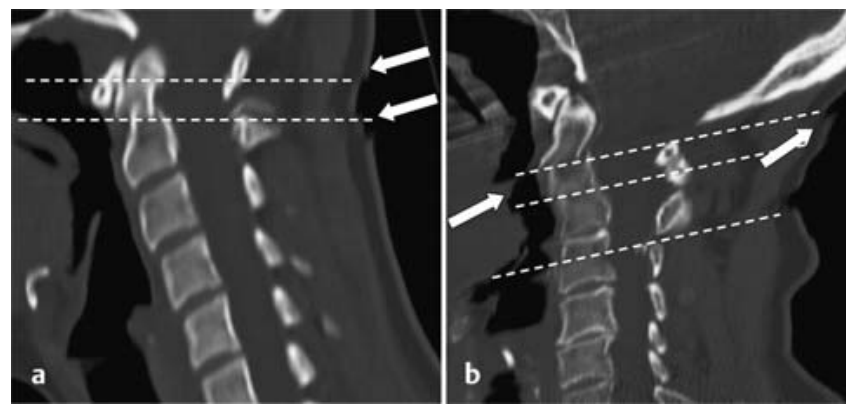

Abb. $9 \mathrm{a}$ und $\mathbf{b}$ Ähnliches Fallbeispiel mit vermeintlicher Densfraktur. Deutlich wird das Artefakt anhand des Pixelversatzes auf Hautniveau (Hilfslinien).

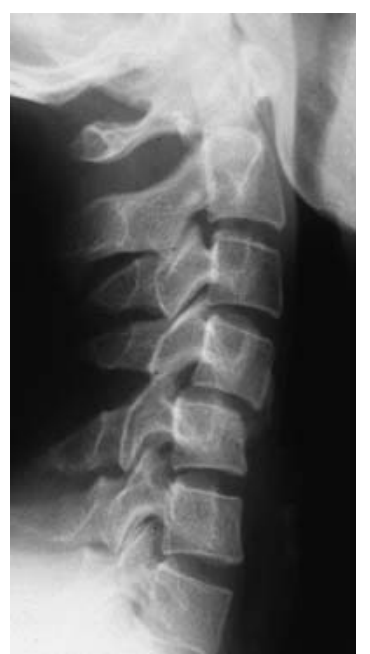

Abb. 10 Die Nativdiagnostik zeigt den kyphotischen Knick in C4/5.

Die MRT dient auch dazu, Verletzungen der dorsalen Strukturen auszuschließen, um eine rein ventrale Stabilisierung zu planen.

Der Nachteil der MRT ist sicherlich die deutlich niedrigere Knochenspezifität, sodass die CT-Untersuchung weiterhin

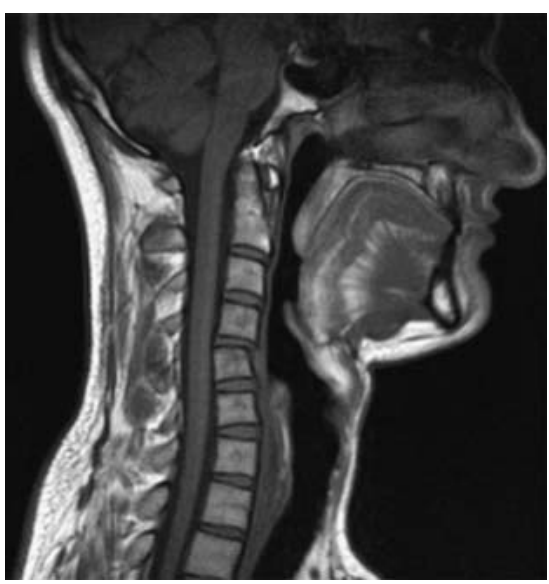

Abb. 11 Die MRT-Diagnosik zeigt in der T1Wichtung die Konturunterbrechung des vorderen Längsbands und somit die Instabilität.

der Goldstandard bleibt bei V.a. Verletzungen im HWS-Bereich. Die Sensitivität der MRT ist im Vergleich zur CT sehr hoch, v.a. im Nachweis eines Bone Bruise. Hierbei sind sog. fettunterdrückte STIRSequenzen zu fordern. Diese Spezialsequenzen sind auch im Nachweis einer Spondylodiszitis und Spondylitis sehr sensitiv (Abb. 14). Zur Planung der Versorgung, insbesondere auch zur Schrau- 


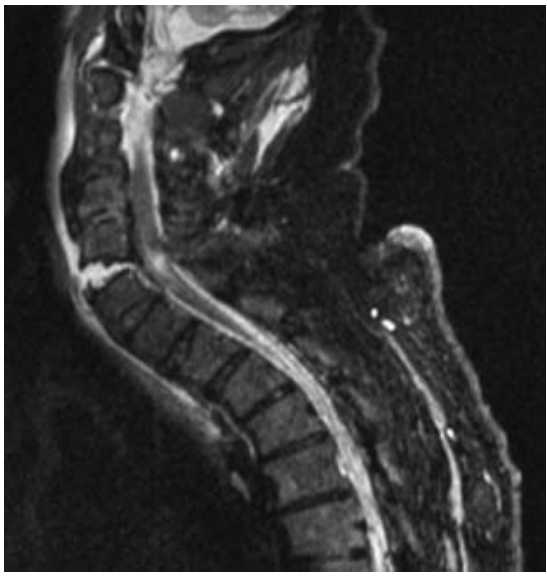

Abb. 12 MRT-Diagnosik einer kompletten BS-Zerreißung beim Bechterew-Patienten. Einblutung und Aufklappen des Bandscheibenfachs mit ventraler Einblutung sowie deutliches Myelonödem.

benplatzierung, ist eine CT-Untersuchung jedoch erforderlich.

Eine weitere Indikation ist der Ausschluss einer Myelomalazie. Ist ein Patient zur operativen Versorgung vorgesehen (insbesondere bei spinaler Dekompression), so sollte schon aus juristischen Gründen eine MRT-Diagnostik erfolgen, um eine evtl. bereits vorliegende Myelomalazie nachzuweisen und darzustellen. Geschieht dies erst nach operativer Versorgung, so bleibt die Frage offen, zu welchem Zeitpunkt die Schädigung eingetreten ist.

Eine weitere Ergänzung der MRT-Diagnostik ist die Gabe von Kontrastmittel. Einerseits können Gefäßdarstellungen erfolgen (MR-Angio). Bei knöchernen Verletzungen des Foramen transversarium können so Verletzungen der Vertebralarterien ausgeschlossen werden. Ebenso können Dissektionen der Karotiden nachgewiesen werden.

Andererseits ist eine Indikation für die Gabe von Kontrastmittel die bessere Einschätzung von Narbengewebe. Insbesondere bei der Unterscheidung Rezidivprolaps gegenüber Narbengewebe nach vorangegangener Sequestrektomie ist die Gabe von KM hilfreich (Anreicherung im Narbengewebe).

Darüber hinaus im Nachweis von septischen Prozessen und epiduralen Abszessen.

Eine Neuerung in der Diagnosik ist das „upright“-MRT. In einer offenen Röhre können dynamische Untersuchungen

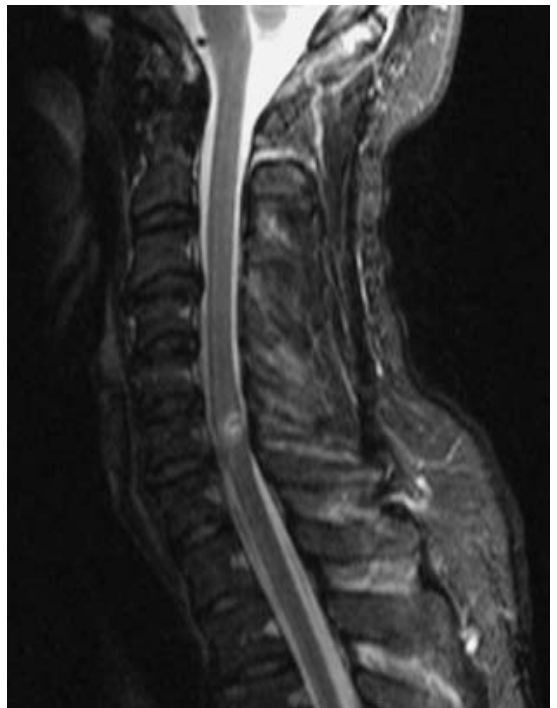

Abb.13 SCIWORA: Myelonödem ohne Zeichen einer ligamentären oder knöchernen Verletzung in diesem Bereich.

durchgeführt werden. So können die Bandscheiben und Neuroforamina unter Last (liegen gegenüber sitzen) wie auch in Reklination und Inklination dargestellt werden. Eine erst in der aufrechten Position nachweisbare foraminale Stenose bzw. funktionelle Stenose wird so offensichtlich. Funktionsuntersuchungen können durchgeführt werden, um sowohl die Stabilität als auch die Auswirkungen auf die Enge des Spinalkanals bei Listhesen darzustellen.

\section{Funktionelle Untersuchungen}

Goldstandard bleibt jedoch weiterhin die nativradiologische funktionelle Untersuchung. Die Hauptindikation stellen diskoligamentäre Verletzungen dar. Besonders an der HWS sind diese häufig und entgehen der Standarddiagnostik meistens. Nach Eintreten der Läsion kommt es durch reflektorische Anspannung der Nackenmuskulatur zur Spontanreposition und die Schädigung entgeht dem Nachweis auf der Übersichtsaufnahme.

Bei wachen Patienten muss die passive Funktionsuntersuchung durch den Operateur selbst erfolgen. Es ist insbesondere zu eruieren, ob der Patient den Bewegungsablauf mitmachen kann oder aufgrund von Schmerzen dem entgegenarbeitet. Der Kopf des in Rückenlage befindlichen Patienten wird vom Untersucher mit beiden Händen geführt (Abb. 15). Er wird dann unter seitlicher Durchleuchtung in maximale Reklination und Inklination gebracht.

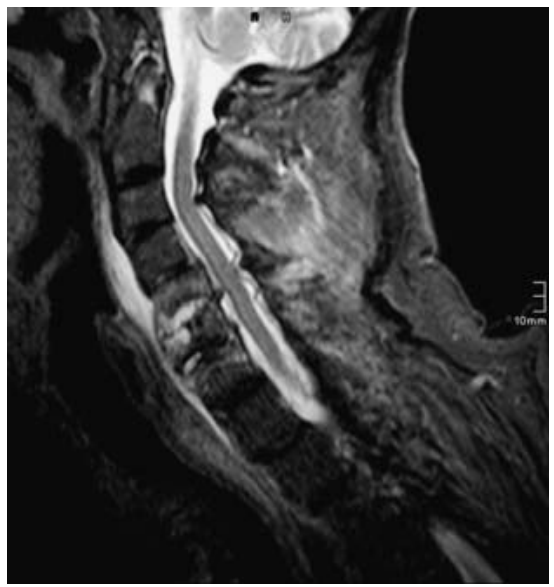

Abb. 14 Spondylodiszitis mit Spondylitis und Inhibierung der ventralen Halsweichteile in der STIR-Sequenz.

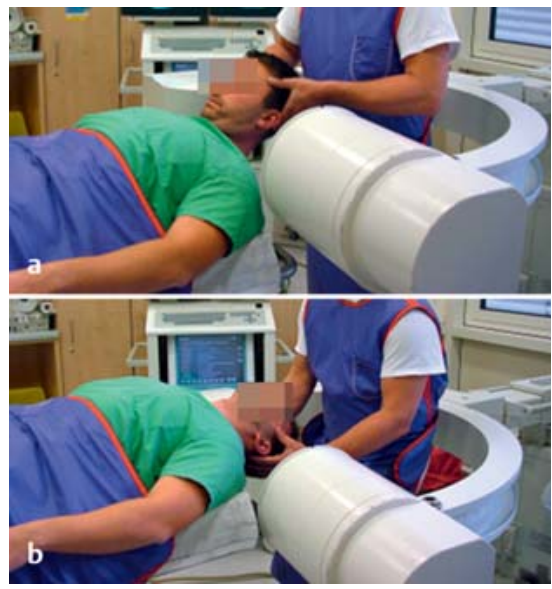

Abb. 15a und b Funktionsuntersuchung in Inklination und Reklination durch geführte Bewegung im seitlichen Strahlengang.

Ein Gleitvorgang zweier Wirbelkörper zueinander bzw. ein ventrales Aufklappen sind Zeichen der Instabilität (Abb. 16).

Auch hier sind die Problemzonen der kraniozervikale und der zervikothorakale Übergang. Eine Erweiterung der funktionellen Diagnosik ist die CT-Darstellung in Inklination und Reklination. Für die Stabilität von Denspseudarthrosen ist eine Indikation gegeben (Abb. 17), weiterhin für Instabilitäten im Bereich C7/Th1. Eine Indikation ergibt sich hierbei nicht nur bei traumatischen Läsionen, sondern auch bei degenerativen Instabilitäten und Anschlussinstabilitäten bei darüberliegenden Fusionen (Abb. 18).

\section{Myelo-CT}

Die Myelo-CT-Untersuchung mit Funktionsaufnahmen hat ihren Stellenwert im degenerativen Spektrum. Insbeson- 


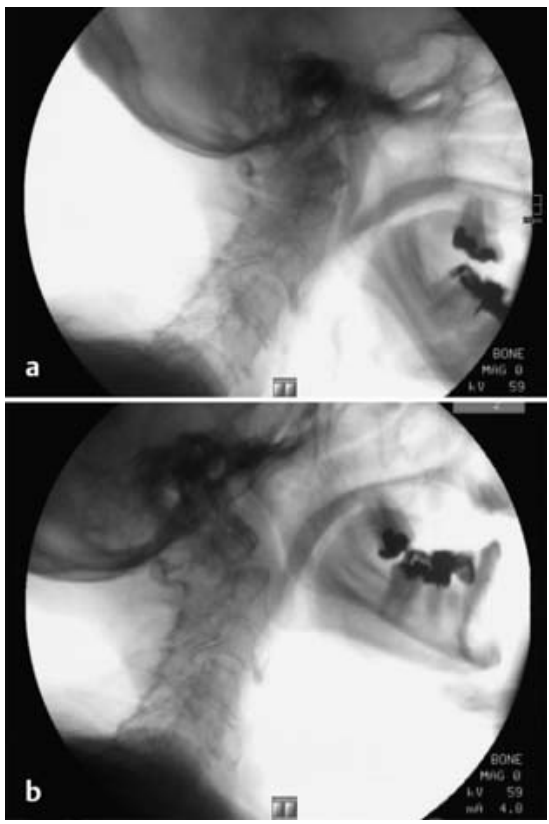

Abb. 16 a und b Funktionsuntersuchung des Dens axis mit erheblicher Instabilität in Inklination und Reklination.

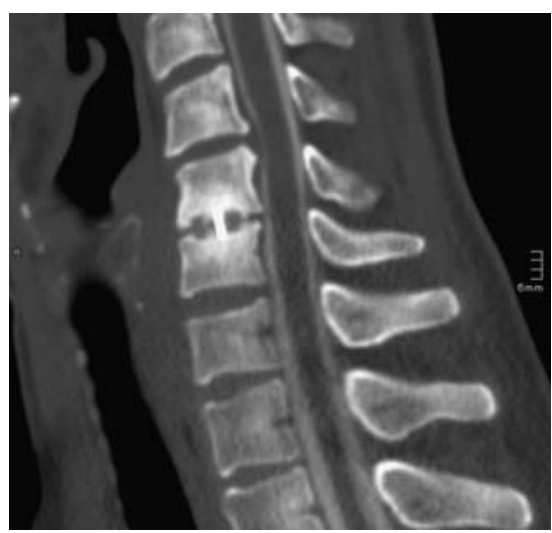

Abb. 19 Relative spinale Enge im Myelo-CT im kranialen Anschlussegment nach Fusion mit noch erhaltenem Liquor/KM-Saum um die Dura.

dere bei mehrsegmentalen spinalen Engen ist hierfür eine Indikation gegeben, um die OP-Planung auszuarbeiten. Instabilitäten sind dabei im Rahmen der Funktionsaufnahmen darstellbar. Nach intrathekaler KM-Applikation wird die CT-Schichtung nach $2 \mathrm{~h}$ durchgeführt, um eine ausreichende Verteilung des KM zu gewährleisten. Schrägaufnahmen sind in diesem Fall sinnvoll, um die Neuroforamina und Wurzeltaschen darzustellen.

Die bevorzugte Indikation sind auch hier Anschlussinstabilitäten nach vorangegangener Fusion (Abb. 19), insbesondere bei einliegenden Metallimplantaten. Diese führen bei der MRT-Diagnostik be-
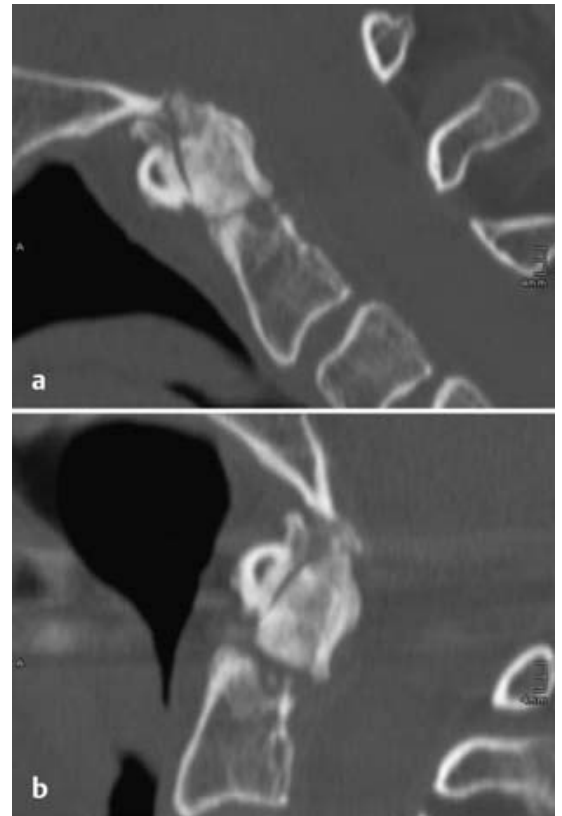

Abb. 17a und b CT-Funktionsuntersuchung des Dens axis bei Pseudarthrose mit leichter Instabilität.
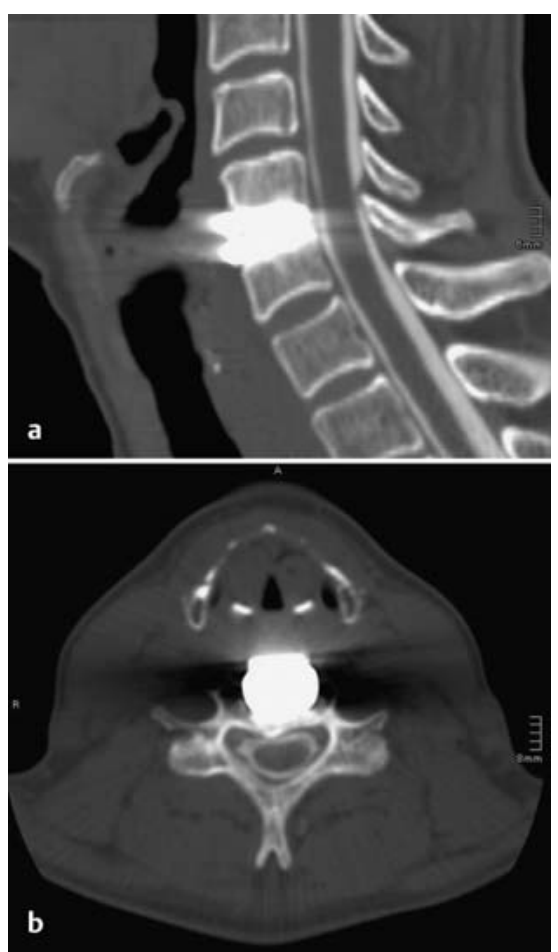

Abb. 20 a und b Bei einliegenden Bandscheibenprothesen zeigt die Myelo-CT-Darstellung eine ausreichende Genauigkeit angesichts der spinalen Weite. In diesem Fall besteht eine mediolaterale rechtsseitige Spondylose hinter der Prothese.

kanntermaßen zur Artefaktauslöschung im dahinterliegenden Spinalkanal. Ein Beispiel sind hierfür die in letzter Zeit überhandnehmenden Implantationen von Bandscheibenprothesen. Die Dar-

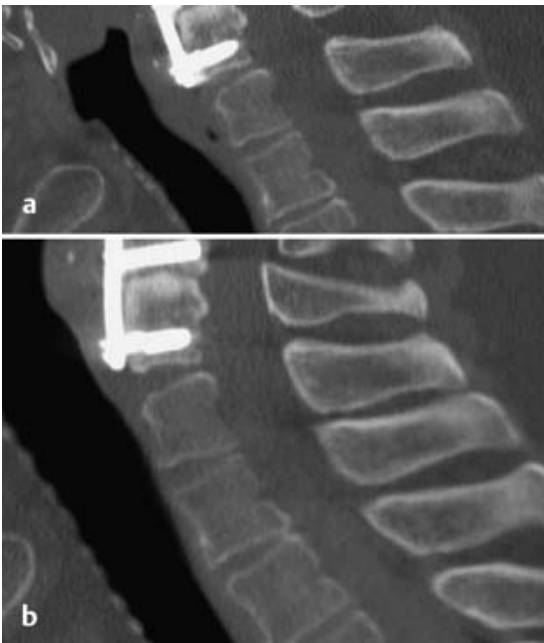

Abb. 18a und b CT-Funktionsuntersuchung des zervikothorakalen Übergangs bei Anschlussinstabilität nach Fusion mit kaudalem Schraubenbruch. Nach ausgeschöpfter konservativer und infiltrativer Therapie erfolgte die Erweiterung der Fusion auf Th1.

stellbarkeit leidet hierbei erheblich (Abb. 20). Solche Patienten erscheinen leider zu oft in den Ambulanzen bei weiterhin bestehendem Leidensbild.

Eine Indikation ist ebenso gegeben bei Kontraindikationen zur MRT-Diagnostik, wie z.B. einliegenden Herzschrittmachern.

\section{Lagerungstechniken}

In Abhängigkeit des Dislokationsgrads und der Verletzungsschwere sind verschiedene Repositionshilfen und Lagerungshilfen vorrätig.

Generell gilt, dass bei verhakten Luxationen hohe Kräfte aufgewendet werden müssen, um eine suffiziente Reposition zu erreichen. Hierzu hat sich ein HWSAggregat als sehr hilfreich erwiesen (Abb. 21). Der Kopf des Patienten wird in einen Halo-Ring eingespannt. Ähnlich wie bei einem Extensionstisch zur Reposition von proximalen Femurfrakturen können hohe Zugkräfte in Längsrichtung angewandt werden. Ein Hypomochlion kann im Bereich des Nackens einwirken. Weiterhin können die Inklination und Reklination sowie die Rotation stufenfrei eingestellt werden (s. Pfeile Abb. 21). Die Verbindungsstreben sind dabei so angebracht, dass ein biplanares Röntgen in beiden Ebenen simultan möglich ist (Abb. 22). Das Repositionsgerät kann sowohl in Bauch- als auch in Rückenlage angebracht werden. Sollte selbst hiermit eine verhakte Luxation nicht reponibel 

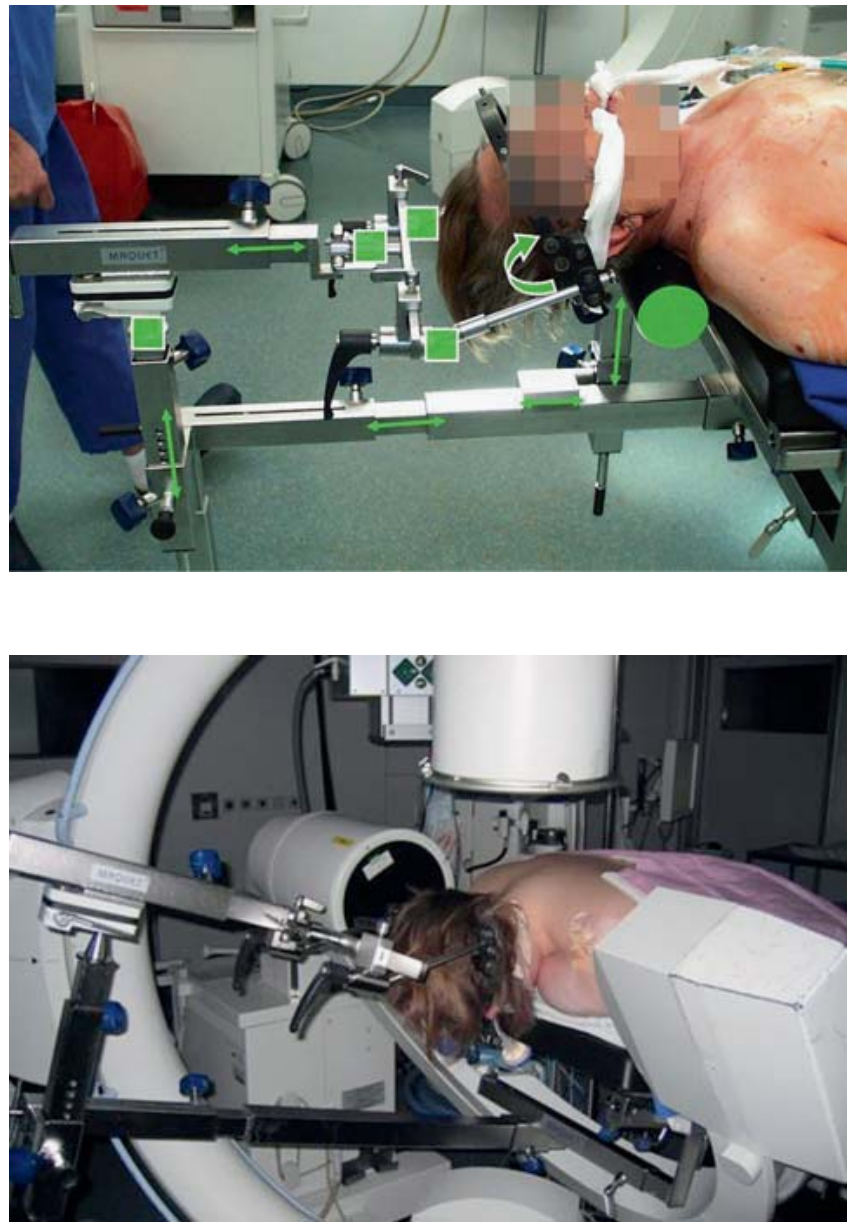

Abb. 22 Die Instrumentierung sollte mit 2 BW-Einheiten erfolgen, um Schwierigkeiten beim Durchschwenken zu vermeiden. Die Durchleuchtung gelingt dabei überlagerungsfrei.

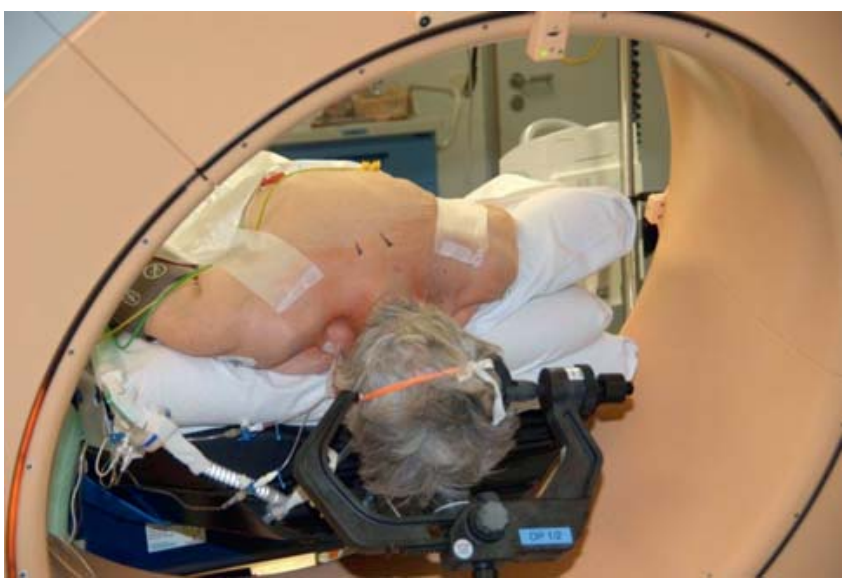

Abb. 23 Lagerung in einer Karbon-Mayfield-Klemme im geschlossenen O-Arm.

sein, so bleibt nur die offene Repostion mit Abtragung der Gelenkfacette und Dekompression mit ggf. dorsoventralem Vorgehen.

Für einfachere Frakturen kann die Lagerung mit einer Mayfield-Klemme angewandt werden. Auch hier wird der HaloRing in einen Klemmmechanismus stufenfrei unter sicherer Fixation eingespannt. Hohe Zugkräfte können dabei nicht angewandt werden, jedoch gelingt es, eine maximale Inklination zu halten.

Ergänzend besteht die Möglichkeit, eine Karbon-Mayfield zu verwenden, um z.B. eine überlagerungsfreie Darstellung bei 3-dimensionalen Rekonstruktionen und Schichtaufnahmen zu erhalten. Weiterhin ist hierfür ein Karbontisch erforderlich. Insbesondere für dorsale C2/1-Verschraubungen in navigierter Technik bzw. mit intraoperativer CT-Kontrolle

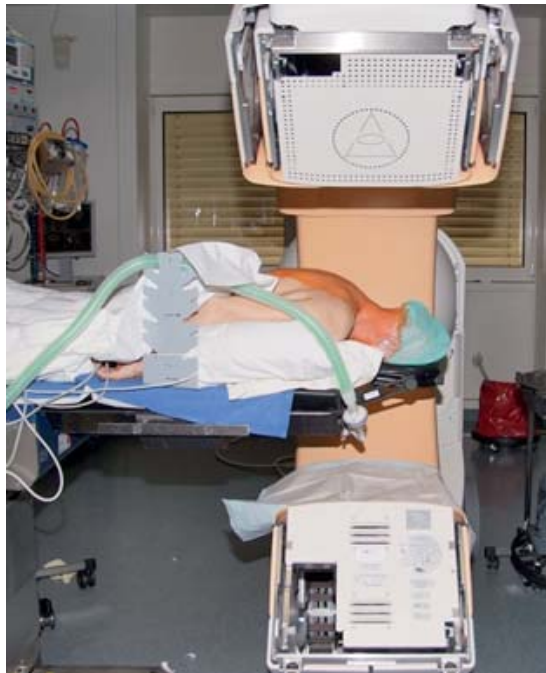

Abb. 24 Lagerung in einer Karbonkopfschale im geöffneten O-Arm-Ring beim Einfahren.

hat sich die Karbon-Mayfield bewährt (Abb. 23). Es werden dabei Einmalpins aus Kunststoff verwandt.

Für einfachere Lagerungstechniken genügt eine Karbonkopfschale, hierbei bestehen jedoch erhebliche Einschränkungen angesichts einer stabilen Lagerung in Inklination oder Reklination (Abb. 24). Meist wird diese Lagerungstechnik für Standardeingriffe von ventral verwandt (Nukleotomie etc.). Der Kopf sollte dabei zusätzlich mit einem Pflasterzügel gesichert werden. Weiterhin werden die Schultern durch Pflasterzügel mit Zugrichtung nach kaudal geklebt, um eine bessere Darstellbarkeit im zervikothorakalen Übergang zu erreichen.

\section{Invasive Diagnostik}

Bei degenerativen Erkrankungen ist bei fehlenden fokalneurologischen Defiziten eine konservative Therapie voranzustellen. Bei Beschwerdepersistenz mit immobilisierenden Schmerzen ist die weitere invasive Therapie zu planen. Hierzu zählen zunächst Infiltrationsmaßnahmen. Im traumatologischen Patientengut sind diese meist nicht erforderlich.

\section{Facetteninfiltration}

Die Infiltration der kleinen Wirbelgelenke zeigt eine weite Verbreitung. Bei gutem Ansprechen kann eine Vereisung bzw. Thermotherapie angeschlossen werden. Sie ist dann auch ein Ausschlusskriterium für die Bandscheibenprothetik. 


\section{Spinalwurzelinfiltration}

Bei neuroforaminalen Engen mit schmerzhaften, radikulär zuordenbaren Dysästhesien kann eine Nervenwurzelbetäubung einen guten Behandlungserfolg erzielen. Bei positivem Infiltrationsergebnis sollte mit gezielter Cortisoninfiltration weiterbehandelt werden. Nur bei rezidivierenden Beschwerden kann die operative Dekompression indiziert werden.

\section{Diskografie}

Der diskogene Rückenschmerz ist klinisch schwer zu erfassen. Bei Verdacht kann eine sog. Provokationsdiskografie mit positivem „memory-pain“ einen Anhalt hierfür geben. Die Rate falsch positiver Befunde beträgt jedoch über $20 \%$. Die Sensitivität kann erhöht werden durch ein gesundes Referenzbandscheibenfach. Weiterhin durch abschließende Injektion eines Lokalanästhetikums mit dann wieder nachlassendem „memory-pain“.

\section{Neurophysiologische Diagnostik}

Bei klinischem Nachweis von Paresen bzw. radikulär fassbaren Dysästhesien sollte eine Elektrophysiologie zur Fragestellung einer floriden Denervation durchgeführt werden. Bei akut auftretender Symptomatik entgeht jedoch der Nachweis, diese sind nach frühestens 2-3 Wochen erfassbar. Die floride Denervation wird in der Nadel-EMG-Untersuchung verifiziert.

Im Unterschied hierzu wird eine Leitungsverzögerung im Rahmen einer SEP-Untersuchung dargestellt. An den unteren Extremitäten wird diese am sinnvollsten durch Tibialis-SEPs, an den oberen anhand von Medianus-SEPs dargestellt. Sie stellt den elektrophysiologisch messbaren Befund der Myelomalazie dar. Klinisch sind hierzu gesteigerte Reflexe und eine Gangataxie passend.

\section{Kontrolle bei HWS-Eingriffen}

Die Anatomie der HWS mit ihren diffizilen und grazilen knöchernen Strukturen erfordert einerseits ein präzises chirurgisches Handwerk als auch andererseits eine exakte Kontrolle und Überprüfung im OP.

Insbesondere für Fusionen und Verschraubungen im Bereich der oberen HWS ist die native Röntgendiagnostik mittels C-Arm oft ungenau [10]. Eine
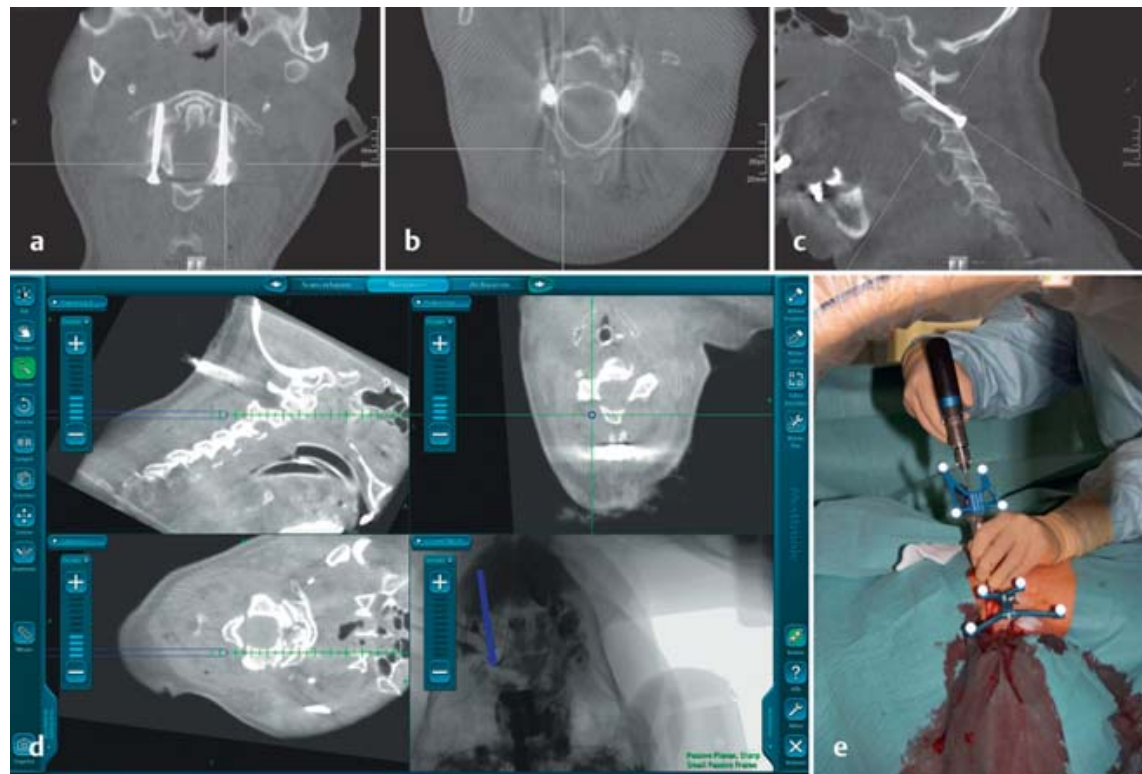

Abb. 25a bis e Qualität der heutigen intraoperativen Schnittbildgebung am Beispiel der perkutanen dorsalen C2/C1-Verschraubung. Eine exakte Lagebeurteilung der Schrauben ist gewährleistet. Die navigierbaren Schnittbildebenen können frei gewählt werden, Trajektorien dienen der Orientierung. Die Schraubenplatzierung gelingt über navigierbare Bohrhülsen.

Neuerung und Erweiterung der Genauigkeit ergibt sich durch die Möglichkeiten der Navigation sowie der CT-Darstellung im OP. Die pedikuläre Schraubenplatzierung an der HWS ist im Wesentlichen erst durch die Navigation ermöglicht worden. Weitere Verbesserungen sind die Möglichkeiten der perkutanen Techniken. Insbesondere die C2/1-Instrumentierung wird erst durch die Schnittbilddiagnostik im OP ermöglicht sowie exakter in ihrer Anwendung (Abb. 25).

Anwendungsgebiet der Schnittbilddiagnostik ist z.B. die Kontrolle des Repositionsergebnisses. Verrenkungsbrüche der oberen HWS sind meist auch mit einer Rotationsfehlstellung im C1/2-Gelenk verbunden, welche 2-dimensional schwer darzustellen ist. Die intraoperative Schnittbilddiagnostik mit sagittaler und koronarer Rekonstruktion zeigt hier eine deutlich höhere Präzision. Dies gilt v.a. für die im Alter häufig auftretenden C2/1-Rotationsfehlstellungen. Insuffiziente Repositionsergebnisse können so direkt intraoperativ optimiert werden, ohne dass ein 2-zeitiges Vorgehen zur Korrektur nach postoperativer CT-Darstellung erforderlich wird.

Die Möglichkeiten der Navigation eröffnen ganz neue Instrumentierungsmöglichkeiten. Die pedikuläre Schraubenplatzierung wie auch die Gelenkschrauben C2/1 und Verschraubungen nach Judet des C2-Bogens werden in ihrer Anwen- dung deutlich sicherer. Ein weiterer Vorteil ist die strahlungsarme Anwendbarkeit. Im Falle der „stealth station“ und des „O-Arms“ entfällt auch die Notwendigkeit, präoperative Planungs-CTs mit Markern anzufertigen. Es wird hierbei im OP nahe des Operationsgebiets ein Tracker platziert zur optoelektronischen Erkennung. Die Daten der Schnittbilddiagnotik mit Marker werden mit der „stealth station“ gematched. Die Navigation gelingt dann über optoelektronisch gesteuerte Instrumente und Darstellung derselben über Trajektorien in der Schnittbilddiagnostik. Eine weitere radiologische Überprüfung ist dann nicht mehr erforderlich. Abschließend sollte jedoch ein CT zur Validierung durchgeführt werden. Rekonstruktionen können nach eigenem Anspruch angefertigt werden und die passenden Ebenen können entprechend des Schraubenverlaufs gedreht werden (Abb. 25).

\section{Literatur}

\footnotetext{
${ }^{1}$ Aebi M, Mohler J, Zach GA et al. Indication, surgical technique, and results of 100 surgicallytreated fractures and fracture-dislocations of the cervical spine. Clin Orthop Relat Res 1986; 203: 244-257

2 Aebi M, Nazarian S. [Classification of injuries of the cervical spine]. Orthopaede 1987; 16 : 27-36

3 Blauth M, Tscherne H. Grundlagen der Wirbelsäulentraumatologie, Obere HWS, Untere HWS. Unfallchirurgie. Wirbelsäule. Berlin, Heidelberg, New York: Springer; 1998
} 
${ }^{4}$ Ebraheim NA, Haman ST, Xu R et al. The anatomic location of the dorsal ramus of the cervical nerve and its relation to the superior articular process of the lateral mass. Spine 1998; 23: 1968-1971

${ }^{5}$ Effendi B, Roy D, Cornish B et al. Fractures of the ring of the axis. A classification based on the analysis of 131 cases. J Bone Joint Surg [Br] 1981; 63: 319-327

${ }^{6}$ Glaser JA, Jaworks BA, Cuddy BG et al. Variation in surgical opinion regarding management of selected cervical spine injuries - a preliminary study. Spine 1998; 23: 975-984

7 Graham JJ. Complications of cervical spine surgery. A five-year report on a survey of the membership of the cervical spine. Research Society by the Morbity and Mortality Committee. Spine 1989; 14: 1046-1050

${ }^{8}$ Hofmeister M, Potulski M, Späth K et al. Klinische Ergebnisse der ventralen Fixation von HWS-Verletzungen. Osteo Trauma Care 1998; 6: $112-120$

9 Jónsson Jr H, Bring G, Rauschning Wet al. Hidden cervical spine injuries in traffic accident victims with skull fractures. J Spinal Disord 1991; 4: 251-263
${ }^{10}$ Kast E, Mohr K, Richter H-P et al. Complications of transpedicular screw fixation in the cervical spine. Eur Spine J 2006; 15: 327-334

11 Magerl F, Aebi M, Gertzbein SD et al. A comprehensive classification of thoracic and lumbar injuries. Eur Spine J 1994; 3: 184-201

12 Magerl FP, Seemann PS. Stable posterior Fusion of the Atlas and Axis by transarticular Screw Fixation. In: Kehr P, Weidner A, eds. Cervical Spine I. Berlin, Heidelberg, NewYork, Tokyo: Springer; 1987: 322-327

${ }^{13}$ Ulrich C, Bühren V. Verletzungen der Halswirbelsäule. Orthopädie und Unfallchirurgie up2date 2006; 1: 415-446

${ }^{14}$ Ulrich C, Nothwang J. Biomechanik und Klinik der Spondylodese an der unteren HWS. Technik und Implantate. Orthopäde 1999; 28: 637-650

15 Vecsei V, Fuchs M, Gäbler C. Indikationen zum kombinierten dorsoventralen Vorgehen bei HWS-Verletzungen. Osteo Trauma Care 1998; 6: $121-128$

${ }^{16}$ White 3rd AA, Panjabi MM. Clinical Biomechanics of the Spine. Philadelphia, Toronto: J. B. Lippincott Company; 1990
Dr. med. Thomas Weiß

Oberarzt

Dr. med. Oliver Gonschorek

Leitender Arzt

Dr. med. Stefan Hauck

Oberarzt

Dr. med. Volker Bühren

Chefarzt

Abt. Wirbelsäulenchir./Unfallchir.

BG-Unfallklinik

Prof.-Küntscher-Str. 8

82418 Murnau

tweiss@bgu-murnau.de 